\title{
A Proof for the Existence of Chaos in Diffusively Coupled Map Lattices with Open Boundary Conditions
}

\author{
Li-Guo Yuan ${ }^{1,2}$ and Qi-Gui Yang ${ }^{1}$ \\ ${ }^{1}$ School of Mathematical Sciences, South China University of Technology, Guangzhou 510640, China \\ ${ }^{2}$ Department of Applied Mathematics, South China Agricultural University, Guangzhou 510640, China
}

Correspondence should be addressed to Li-Guo Yuan, liguoychina@gmail.com

Received 2 July 2011; Accepted 13 September 2011

Academic Editor: Recai Kilic

Copyright ( 2011 L.-G. Yuan and Q.-G. Yang. This is an open access article distributed under the Creative Commons Attribution License, which permits unrestricted use, distribution, and reproduction in any medium, provided the original work is properly cited.

We first study how to make use of the Marotto theory to prove rigorously the existence of the Li-Yorke chaos in diffusively coupled map lattices with open boundary conditions (i.e., a highdimensional discrete dynamical system). Then, the recent $0-1$ test for chaos is applied to confirm our theoretical claim. In addition, we control the chaotic motions to a fixed point with delay feedback method. Numerical results support the theoretical analysis.

\section{Introduction}

Extensive research has been carried out to discover complex behaviors of various discrete dynamical systems in the past several decades. However, limited rigorous analysis concerning existence of chaos in high-dimensional discrete dynamical systems has been seen in the literature. Since the 1980s, coupled map lattices (CMLs) as high-dimensional discrete system have caused widespread concern [1]. CMLs as chaotic dynamical system models for spatiotemporal complexity are usually adopted. Spatiotemporal complexity is common in nature, such as biological systems, networks of DNA, economic activities, and neural networks [1]. The complex behaviors of CMLs have been studied extensively [1-16]. These mainly include bifurcation [2], chaos [6,7], chaotic synchronization [4, 8-10], and controlling chaos $[5,11,12]$. However, being able to rigorously prove the existence of chaos in CMLs is an important and open question. A rigorous verification of chaos will provide a theoretical foundation for the researchers to discover the complex behaviors in CMLs. Recently, Li et al. $[13,14]$ theoretically analyzed the chaos in one-way coupled logistic lattice with periodic 
boundary conditions and presented a chaotification method for creating spatiotemporal systems strongly chaotic. Tian and Chen [15] discussed the chaos in CMLs with the new chaos definition in the sense of Li-Yorke. These CMLs with the periodic boundary conditions have been most extensively investigated [1, 2, 4-15]. But, in all of the research so far published, only a few studies have attempted to explore the case of open boundary conditions [16,17]. In this case, it is almost impossible to obtain all eigenvalues of Jacobian matrix of the CMLs. This partially hindered early research in the CMLs with open boundary conditions.

Until now, the rigorous proof of chaos has not yet been studied in diffusively coupled map lattices (DCMLs) with open boundary conditions, which is one important case of CMLs. Inspired by the ideas of $[13,14,18,19]$, we have tried to answer this question. The DCML is as follows $[1,16,17]$ :

$$
x_{n+1}(i)=(1-\epsilon) f\left(x_{n}(i)\right)+\frac{\epsilon}{2}\left[f\left(x_{n}(i-1)\right)+f\left(x_{n}(i+1)\right)\right],
$$

where $n$ is discrete time step and $i$ is lattice point $(i=1,2, \ldots, N ; N$ is the number of the sites in the DCML). $\epsilon \in(0,1)$ is the coupling strength. $x_{n}(i)$ represents the state variable for the $i$ th site at time $n$. Throughout this paper, we adopt open boundary conditions [16, 17]:

$$
\begin{gathered}
x_{n+1}(1)=(1-\epsilon) f\left(x_{n}(1)\right)+\epsilon f\left(x_{n}(2)\right), \\
x_{n+1}(N)=\epsilon f\left(x_{n}(N-1)\right)+(1-\epsilon) f\left(x_{n}(N)\right) .
\end{gathered}
$$

Here each of the lattice points in (1.1) and (1.2) is chosen to be the logistic map $f\left(x_{n}(i)\right)=$ $1-a x_{n}^{2}(i)$, where $a \in(0,2]$ and $x_{n}(i) \in(-1,1)$. The logistic function $f(x)=1-a x^{2}$ is equivalent to the well-known form $g(z)=r z(1-z)$ [20] when the transformations $a=r(r-2) / 4$ and $x=2(2 z-1) /(r-2)$ are taken. This simple quadratic iteration was only completely understood in the late 1990s [21]. When the lattice points are logistic functions, the CMLs generate more rich and complex dynamic behaviours. What is more is that the dynamical behaviors of CMLs may be different from each other when the lattice points are chosen from $f(x)$ and $g(z)$, respectively $[1,2]$.

Based on the Marotto theory $[22,23]$, we prove theoretically the existence of the LiYorke chaos in the DCML (1.1). In the process of proving, the most difficult problem is how to find a snap-back repeller. At the same time, we have exploited different measures such as the chaotic phase, bifurcation diagram, and 0-1 test on time series to confirm our claim of the existence of chaos. The $0-1$ test is a new method to distinguish chaotic from ordered motion. It is more suitable to handle high-dimensional systems and does not require phase space reconstruction. Finally, we control spatiotemporal chaotic motion in the DCML (1.1) to period-1 orbit (fixed point) by delay feedback and obtain the stability conditions of control.

The paper is organized as follows. In Section 2, the Marotto theorem is introduced. In Section 3.1, a mathematically rigorous proof of the Li-Yorke chaos in the DCML (1.1) is examined. In Section 3.2, we show numerical simulation results. In Section 3.3, 0-1 test method is used to verify the existence of chaos. In Section 4, delay feedback control method is adopted to control chaos. In the last section, conclusions are given. 


\section{Marotto Theorem}

Li and Yorke [24] state that the period-three orbit exhibits chaos in one-dimensional discrete interval map. This is the first precise definition of discrete chaos. This classical criterion for chaos is extended to higher-dimensional discrete systems by Marotto [22]. Marotto considered the following $n$-dimensional discrete system:

$$
x_{k+1}=F\left(x_{k}\right), \quad k=0,1,2, \ldots,
$$

where $x_{k} \in R^{n}$ and $F: R^{n} \rightarrow R^{n}$ is continuous. Let $B_{r}(x)$ denote the closed ball in $R^{n}$ of radius $r$ centered at point $x$ and $B_{r}^{0}(x)$ its interior. Also, let $\|x\|$ be the usual Euclidean norm of $x$ in $R^{n}$ [22]. Then, if $F$ is differentiable in $B_{r}(z)$, Marotto claimed that in the following, $A \Rightarrow B$.

(A) All eigenvalues of the Jacobian $D F(z)$ of system (2.1) at the fixed point $z$ are greater than one in norm.

(B) There exist some $s>1$ and $r>0$ such that, for all $x, y \in B_{r}(z),\|F(x)-F(y)\|>$ $s\|x-y\|$.

Marotto thought that, if (A) is satisfied, then (B) can be derived, that is, $\mathrm{F}$ is expanding in $B_{r}(z)$ [22]. But, (A) does not always imply (B) with usual Euclidean norm [25]. Chen et al. [26] first pointed out this problem in the Marotto theorem. During the past decade, several papers tried to fix this error $([19,23,25,26]$ and some references therein).

In 2005, Marotto redefined the definition of snap-back repeller [23]. He pointed out that (A) does imply (B) with some vector norm in $R^{n}$ (which depends on $F$ and $z$ ). See, for example, the discussion by Hirsch and Smale in [27]. However, we still do not know what the vector norm is in specific issues. In the application of the Marotto theorem, we need to find a suitable vector norm. With this special vector norm, (A) implies (B). The correct Marotto theorem is given as follows.

Definition 2.1 (see [23]). Suppose that $z$ is a fixed point of (2.1) with all eigenvalues of $D F(z)$ exceeding 1 in magnitude, and suppose that there exists a point $x_{0} \neq z$ in a repelling neighborhood of $z$, such that $x_{M}=z$ and $\operatorname{det}\left(D F\left(x_{k}\right)\right) \neq 0$ for $1 \leq k \leq M$, where $x_{k}=F^{k}\left(x_{0}\right)$. Then, $z$ is called a snap-back repeller of $F$.

Lemma 2.2 (see [23], the Marotto theorem). If F has a snap-back repeller, then $F$ is chaotic.

At the same time, Shi and Chen [19] presented a modified Marotto theorem as follows.

Lemma 2.3 (see [19]). Consider the n-dimensional discrete system

$$
x_{k+1}=F\left(x_{k}\right), \quad x_{k} \in R^{n}, k=0,1,2, \ldots,
$$

where $F$ is a map from $R^{n}$ to itself. Assume that $F$ has a fixed point $x^{*}$ satisfying $x^{*}=F\left(x^{*}\right)$. Assume, moreover, that

(1) $F(x)$ is continuously differentiable in a neighborhood of $x^{*}$, and all eigenvalues of $D F\left(x^{*}\right)$ have absolute values large than 1 , where $D F\left(x^{*}\right)$ is the Jacobian of $F$ evaluated at $x^{*}$, which implies that there exist an $r>0$ and a norm $\|\cdot\|$ in $R^{n}$ such that $F$ is expanding in $\bar{B}_{r}\left(x^{*}\right)$, the closed ball of radius $r$ centered at $x^{*}$ in $\left(R^{n},\|\cdot\|\right)$, 
(2) $x^{*}$ is a snap-back repeller of $F$ with $F^{m}\left(x_{0}\right)=x^{*}, x_{0} \neq x^{*}$, for some $x_{0} \in B_{r}\left(x^{*}\right)$ and some positive integer $m$, where $B_{r}\left(x^{*}\right)$ is the open ball of radius $r$ centered at $x^{*}$ in $\left(R^{n},\|\cdot\|\right)$. Furthermore, $F$ is continuously differentiable in some neighborhoods of $x_{0}, x_{1}, \ldots, x_{m-1}$, respectively, and $\operatorname{det}\left[D F\left(x_{j}\right)\right] \neq 0$, where $x_{j}=F\left(x_{j-1}\right)$ for $j=1,2, \ldots, m$.

Then, the system (2.2) is chaotic in the sense of Li-Yorke. Moreover, the system (2.2) has positive topological entropy. Here the topological entropy of $F$ is defined to be the supremum of topological entropies of $F$ restricted to compact invariant sets.

Remark 2.4. The Marotto theorem is a sufficient condition for the Li-Yorke chaos. Lemmas 2.2 and 2.3 have the same effect. But, direct application of the Marotto theorem is not always easy. In most cases, the verification must be carried out with the aid of a computer [28].

\section{Proving Chaos and Simulation Verifications}

\subsection{Proving Chaos}

In this subsection, we prove the existence of the Li-Yorke chaos in the DCML (1.1). Lemmas 3.1 and 3.2 will be useful throughout the proof.

Lemma 3.1 (see $[29,30])$. For a matrix $A_{N \times N}$ with eigenvalues $\lambda_{1}, \lambda_{2}, \ldots, \lambda_{N}$, the determinant of $A$ is equal to $\prod_{i=1}^{N} \lambda_{i}$. Denote $\operatorname{det}(A)=\prod_{i=1}^{N} \lambda_{i}$.

Lemma 3.2 (see $[29,30]$, the Gershgorin circle theorem). Let $A$ be an $n \times n$ matrix, and let $R_{i}$ denote the circle in the complex plane with center $a_{i i}$ and radius $\sum_{j=1, j \neq i}^{n}\left|a_{i j}\right|$; that is,

$$
R_{i}=\left\{z \in \mathcal{C}|| z-a_{i i}\left|\leq \sum_{\substack{j=1, j \neq i}}^{n}\right| a_{i j} \mid\right\},
$$

where $\mathcal{C}$ denotes the complex plane. The eigenvalues of $A$ are contained within $R=\bigcup_{i=1}^{n} R_{i}$. Moreover, the union of any $k$ of these circles that do not intersect the remaining $(n-k)$ contains precisely $k$ (counting multiplicities) of the eigenvalues.

Theorem 3.3. If $0<\epsilon<1 / 2$ and $\epsilon$ is small enough, $a \in\{a \mid a>(1+\sqrt{2}) / 2 \simeq 1.2071\} \bigcap\{a \mid a>$ $\left.(1-\epsilon)^{2} /(1-2 \epsilon)^{2}-(1 / 4)\right\}$, and $c=1 / \sqrt{(3 N+2) \epsilon^{2}-4 N \epsilon+2 N}<0.0613 / \sqrt{2}$, then the DCML (1.1) is chaotic in the sense of Li-Yorke.

Proof. We will prove that the DCML (1.1) has a snap-back repeller $\mathbf{x}^{*}$. Rewrite the DCML (1.1) in the vector form as follows:

$$
\mathbf{x}_{k+1}=F\left(\mathbf{x}_{k}\right),
$$

where $\mathbf{x}_{k}=\left[x_{k}(1), x_{k}(2), \ldots, x_{k}(N)\right]^{T}$ and $T$ denotes the vector (or matrix) transpose. Using Definition 2.1 and Lemma 2.3, we have to verify the following three conditions.

(a) $\mathbf{x}^{*}$ is a fixed point of $F$ and all the eigenvalues of $D F\left(\mathbf{x}^{*}\right)$ have absolute values larger than 1. Moreover, there exist $r>0$ and a norm $\|\cdot\|$ in $R^{n}$ such that $F$ is expanding in $\bar{B}_{r}\left(\mathbf{x}^{*}\right)$. 
(b) There exist a $\mathbf{x}_{0} \in B\left(\mathbf{x}^{*}, r\right)$ and $\mathbf{x}_{0} \neq \mathbf{x}^{*}$ such that $F^{m}\left(\mathbf{x}_{0}\right)=\mathbf{x}^{*}$ for some $m \in \mathbb{N}$ and $m \geq 2$.

(c) $\operatorname{det}\left[D F^{m}\left(\mathbf{x}_{0}\right)\right] \neq 0$.

The proof consists of four steps. The ideas are motivated chiefly by [13, 18, 19].

Step 1. Let $\mathbf{x}^{*}=[(\sqrt{4 a+1}-1) / 2 a, \ldots,(\sqrt{4 a+1}-1) / 2 a]^{T}=z^{*} \mathbf{1} \in R^{N}$, where $z^{*}=(\sqrt{4 a+1}-$ $1) / 2 a, \mathbf{1}=[1, \ldots, 1]^{T}$. Then $\mathbf{x}^{*}$ is a fixed point of the DCML (3.2), that is, $\mathbf{x}^{*}=F\left(\mathbf{x}^{*}\right) . F(\mathbf{x})$ is continuously differentiable in $\bar{B}_{r}\left(\mathbf{x}^{*}\right)$ for some $r>0$. Its Jacobian matrix at $\mathbf{x}^{*}$ is

$$
D F\left(\mathbf{x}^{*}\right)=\left(\begin{array}{cccccc}
(1-\epsilon) f^{\prime}\left(z^{*}\right) & \epsilon f^{\prime}\left(z^{*}\right) & 0 & 0 & \cdots & 0 \\
\frac{\epsilon}{2} f^{\prime}\left(z^{*}\right) & (1-\epsilon) f^{\prime}\left(z^{*}\right) & \frac{\epsilon}{2} f^{\prime}\left(z^{*}\right) & 0 & \ldots & 0 \\
0 & \frac{\epsilon}{2} f^{\prime}\left(z^{*}\right) & (1-\epsilon) f^{\prime}\left(z^{*}\right) & \frac{\epsilon}{2} f^{\prime}\left(z^{*}\right) & \ldots & 0 \\
\vdots & \vdots & \vdots & \vdots & \vdots & \vdots \\
0 & 0 & \ldots & \frac{\epsilon}{2} f^{\prime}\left(z^{*}\right) & (1-\epsilon) f^{\prime}\left(z^{*}\right) & \frac{\epsilon}{2} f^{\prime}\left(z^{*}\right) \\
0 & 0 & \cdots & 0 & \epsilon f^{\prime}\left(z^{*}\right) & (1-\epsilon) f^{\prime}\left(z^{*}\right)
\end{array}\right)
$$

where $f^{\prime}\left(z^{*}\right)=1-\sqrt{4 a+1}<0$. We denote $D F\left(\mathbf{x}^{*}\right)$ by $(1-\sqrt{4 a+1}) M$, where

$$
M=\left(\begin{array}{cccccc}
1-\epsilon & \epsilon & 0 & 0 & \cdots & 0 \\
\frac{\epsilon}{2} & 1-\epsilon & \frac{\epsilon}{2} & 0 & \cdots & 0 \\
0 & \frac{\epsilon}{2} & 1-\epsilon & \frac{\epsilon}{2} & \cdots & 0 \\
\vdots & \vdots & \vdots & \vdots & \vdots & \vdots \\
0 & 0 & \cdots & \frac{\epsilon}{2} & 1-\epsilon & \frac{\epsilon}{2} \\
0 & 0 & \cdots & 0 & \epsilon & 1-\epsilon
\end{array}\right)
$$

Obviously, $M$ is not a circulant matrix. When $N$ is large, it will be difficult to calculate all the eigenvalues of the matrix $D F\left(\mathbf{x}^{*}\right)$. With the Marotto theorem (Lemmas 2.2 and 2.3), we do not need to know the size of eigenvalues and only need to know that the absolute value of eigenvalues is greater than one. According to the Gershgorin circle theorem (Lemma 3.2), all the eigenvalues of $D F\left(x^{*}\right), \lambda_{j}(j=1,2, \ldots, N)$, are given by $1-\sqrt{4 a+1} \leq \lambda_{j} \leq(1-\sqrt{4 a+1})(1-$ $2 \epsilon)$. Under the conditions of Theorem 3.3, that is, $0<\epsilon<0.5$ and $a>(1-\epsilon)^{2} /(1-2 \epsilon)^{2}-(1 / 4)$, the following results are obtained:

$$
1<(\sqrt{4 a+1}-1)(1-2 \epsilon) \leq\left|\lambda_{j}\right| \leq \sqrt{4 a+1}-1, \quad \forall j=1,2, \ldots, N,
$$

that is, all the eigenvalues of $D F\left(\mathbf{x}^{*}\right)$ are greater (in absolute value) than one. $\mathbf{x}^{*}$ is an expanding fixed point of $F$. Therefore, there exist some $r>0$ and a special vector norm $\|\cdot\|$ such that $F$ is expanding in $\bar{B}_{r}\left(\mathbf{x}^{*}\right)$. That is, for any two distinct points $\mathbf{x}, \mathbf{y} \in \bar{B}_{r}\left(\mathbf{x}^{*}\right)$, we have

$$
\|F(\mathbf{y})-F(\mathbf{x})\|>s\|\mathbf{y}-\mathbf{x}\|,
$$


where $s>1$ and $\mathbf{x}, \mathbf{y}$ are sufficiently close to $\mathbf{x}^{*}$. Since $F(\mathbf{y})-F(\mathbf{x})=D F(\mathbf{x})(\mathbf{y}-\mathbf{x})+\alpha$, where $\|\alpha\| /\|\mathbf{y}-\mathbf{x}\| \rightarrow 0$ as $\|\mathbf{y}-\mathbf{x}\| \rightarrow 0$ [19], specially, $\left\|F(\mathbf{x})-F\left(\mathbf{x}^{*}\right)\right\|=\left\|D F\left(\mathbf{x}^{*}\right)\left(\mathbf{x}-\mathbf{x}^{*}\right)+\alpha\right\|$. When $\varepsilon$ is small enough, we can prove that the operator $D F\left(\mathbf{x}^{*}\right)$ is expanding with Frobenius matrix norm $\|\cdot\|_{F}$, where $\|D F(\mathbf{x})\|_{F}=\left(\sum_{j=1}^{N} \sum_{i=1}^{N} a_{i j}^{2}\right)^{1 / 2}$. With the conditions of Theorem 3.3, we get $|(1-\sqrt{4 a+1})(1-\epsilon)|>1$. For any point $\mathbf{x} \in B_{r}\left(\mathbf{x}^{*}\right)$ and $\epsilon$ small enough, there exists some $s>1$ such that

$$
\begin{aligned}
\left\|D F\left(\mathbf{x}^{*}\right) \mathbf{x}\right\|_{F} & =\left\|(1-\sqrt{4 a+1}) M\left(x_{1}, x_{2}, x_{3}, \ldots, x_{N-2}, x_{N-1}, x_{N}\right)^{T}\right\|_{F} \\
& =\left\|(1-\sqrt{4 a+1})\left(\begin{array}{c}
(1-\epsilon) x_{1}+\epsilon x_{2} \\
\frac{\epsilon}{2} x_{1}+(1-\epsilon) x_{2}+\frac{\epsilon}{2} x_{3} \\
\vdots \\
\frac{\epsilon}{2} x_{N-2}+(1-\epsilon) x_{N-1}+\frac{\epsilon}{2} x_{N} \\
\epsilon x_{N-1}+(1-\epsilon) x_{N}
\end{array}\right)\right\|_{F} \geq s\left\|\left(\begin{array}{c}
x_{1} \\
x_{2} \\
\vdots \\
x_{N-1} \\
x_{N}
\end{array}\right)\right\|_{F} .
\end{aligned}
$$

Since $F(\mathbf{x})$ is continuously differentiable, $D F(\mathbf{x})$ is also expanding for $\mathbf{x} \in B_{r}\left(\mathbf{x}^{*}\right)$. Let the bound of the maximal open expanding ball $B_{r}\left(\mathbf{x}^{*}\right)$ be denoted by $\rho \mathbf{1}$, where $\rho$ satisfies the following inequality [13]:

$$
\|D F(\rho \mathbf{1})\|=\sqrt{4 a^{2}(1-\epsilon)^{2} N \rho^{2}+2 \epsilon^{2} a^{2}(N-2) \rho^{2}+8 \epsilon^{2} a^{2} \rho^{2}}>1 .
$$

Moreover, the equation

$$
\sqrt{2 a^{2} \rho^{2}\left[(3 N+2) \epsilon^{2}-4 N \epsilon+2 N\right]}=1
$$

has two solutions

$$
\rho_{1,2}=\mp \frac{1}{\sqrt{2} a \times \sqrt{(3 N+2) \epsilon^{2}-4 N \epsilon+2 N}}=\mp \frac{c}{\sqrt{2} a}
$$

where $c=1 / \sqrt{(3 N+2) \epsilon^{2}-4 N \epsilon+2 N}$. One has $c \in(0,1)$ (because $f(\epsilon)=(3 N+2) \epsilon^{2}-4 N \epsilon+$ $2 N$ is a quadratic function, the discriminant $\Delta=-8 N^{2}-16 N<0$, when $\epsilon=4 N / 2(3 N+2)$, $\left.\min f(\epsilon)=\left(4(3 N+2) 2 N-16 N^{2}\right) / 4(3 N+2)>1\right)$. In fact, $c \in(1 / \sqrt{2 N}, 1 / \sqrt{(3 / 4) N+(1 / 2)})$. Since $a>(1+\sqrt{2}) / 2 \approx 1.2071$ and $0<\rho_{2}<z^{*}<1$, we take $\rho=\rho_{2}=c / \sqrt{2} a$. Then, $z^{*}-\rho<1-z^{*}$, and we denote

$$
r=z^{*}-\rho=\frac{\sqrt{4 a+1}-1-\sqrt{2} c}{2 a}>0
$$

Thus, condition (a) of Definition 2.1 and Lemma 2.2 is satisfied. 
Step 2. For all $\mathbf{z}=z \mathbf{1} \in B_{r}\left(\mathbf{x}^{*}\right)$, we have $\left|z-z^{*}\right|<r$, that is, $\sigma_{1}<z<\sigma_{2}$, where $\sigma_{1}=z^{*}-r=$ $c / \sqrt{2} a, \sigma_{2}=z^{*}+r=(\sqrt{4 a+1}-1-(\sqrt{2} / 2) c) / a$. Now let $\mathbf{x}=\left(x_{1}, x_{2}, \ldots, x_{N}\right)^{T}$ and $F(\mathbf{x})=\mathbf{x}^{*}$, that is,

$$
\begin{gathered}
(1-\epsilon)\left(1-a x_{1}^{2}\right)+\epsilon\left(1-a x_{2}^{2}\right)=z^{*}, \\
(1-\epsilon)\left(1-a x_{i+1}^{2}\right)+\frac{\epsilon}{2}\left(1-a x_{i}^{2}\right)+\frac{\epsilon}{2}\left(1-a x_{i+2}^{2}\right)=z^{*}, \\
\epsilon\left(1-a x_{N-1}^{2}\right)+(1-\epsilon)\left(1-a x_{N}^{2}\right)=z^{*}
\end{gathered}
$$

where $i=1,2, \ldots, N-2$. Summing all the above equations, we obtain

$$
\begin{aligned}
\left(1-\frac{\epsilon}{2}\right) & \left(1-a x_{1}^{2}\right)+\left(1+\frac{\epsilon}{2}\right)\left(1-a x_{2}^{2}\right)+\sum_{k=3}^{N-2}\left(1-a x_{k}^{2}\right) \\
+ & \left(1+\frac{\epsilon}{2}\right)\left(1-a x_{N-1}^{2}\right)+\left(1-\frac{\epsilon}{2}\right)\left(1-a x_{N}^{2}\right)=N z^{*}
\end{aligned}
$$

Assume that (3.12) has a solution, and denote $\mathbf{y}_{1}=z_{1} \mathbf{1}$, that is $N\left(1-a z_{1}^{2}\right)=N z^{*}$, which has two solutions: $z_{1}= \pm \sqrt{\left(1-z^{*}\right) / a}= \pm(\sqrt{4 a+1}-1) / 2 a$. We choose $z_{1}=(1-\sqrt{4 a+1}) / 2 a$ since $z_{1}-\sigma_{1}=(1-\sqrt{4 a+1}-\sqrt{2} c) / 2 a<0$, that is, $z_{1}<\sigma_{1}$ and $z_{1} \notin\left(\sigma_{1}, \sigma_{2}\right)$.

Step 3. Now, let $F(\mathbf{x})=\mathbf{y}_{1}$, that is,

$$
\begin{gathered}
(1-\epsilon)\left(1-a x_{1}^{2}\right)+\epsilon\left(1-a x_{2}^{2}\right)=z_{1}, \\
(1-\epsilon)\left(1-a x_{i+1}^{2}\right)+\frac{\epsilon}{2}\left(1-a x_{i}^{2}\right)+\frac{\epsilon}{2}\left(1-a x_{i+2}^{2}\right)=z_{1}, \\
\epsilon\left(1-a x_{N-1}^{2}\right)+(1-\epsilon)\left(1-a x_{N}^{2}\right)=z_{1},
\end{gathered}
$$

where $i=1,2, \ldots, N-2$. Summing the above $N$ equations, we get

$$
\begin{aligned}
(1- & \left.\frac{\epsilon}{2}\right)\left(1-a x_{1}^{2}\right)+\left(1+\frac{\epsilon}{2}\right)\left(1-a x_{2}^{2}\right)+\sum_{k=3}^{N-2}\left(1-a x_{k}^{2}\right) \\
& +\left(1+\frac{\epsilon}{2}\right)\left(1-a x_{N-1}^{2}\right)+\left(1-\frac{\epsilon}{2}\right)\left(1-a x_{N}^{2}\right)=N z_{1} .
\end{aligned}
$$

Assume that the system of (3.14) has a solution, and denote $\mathbf{y}_{2}=z_{2} \mathbf{1}$, that is, $N\left(1-a z_{2}^{2}\right)=N z_{1}$, that is, $1-a z_{2}^{2}=(1-\sqrt{4 a+1}) / 2 a$, which has two solutions: $z_{2}= \pm \sqrt{(2 a+\sqrt{4 a+1}-1) / 2 a^{2}}$. We take $z_{2}=\sqrt{(2 a+\sqrt{4 a+1}-1) / 2 a^{2}}$. Thus,

$$
\begin{aligned}
\sigma_{2}-z_{2} & =\frac{\sqrt{4 a+1}-1-(\sqrt{2} / 2) c}{a}-\sqrt{\frac{2 a+\sqrt{4 a+1}-1}{2 a^{2}}} \\
& =\frac{\sqrt{8 a+2}-\sqrt{2}-c-\sqrt{2 a-1+\sqrt{4 a+1}}}{\sqrt{2} a} .
\end{aligned}
$$


Denote $\sqrt{4 a+1}=t$; since $a>(1+\sqrt{2}) / 2$, that is, $3>t>\sqrt{3+2 \sqrt{2}} \approx 2.4142$ and $a=\left(t^{2}-1\right) / 4$, then

$$
\begin{aligned}
\sqrt{8 a+2}-\sqrt{2}-c-\sqrt{2 a-1+\sqrt{4 a+1}} & =\sqrt{2} t-\sqrt{2}-c-\sqrt{\frac{t^{2}-1}{2}+t-1} \\
& =\frac{2 t-\sqrt{t^{2}+2 t-3}-2-\sqrt{2} c}{\sqrt{2}} .
\end{aligned}
$$

Denoting $y(t)=2 t-\sqrt{t^{2}+2 t-3}$, we get $y^{\prime}(t)=2-(t+1) /\left(\sqrt{(t+1)^{2}-4}\right)>0, t \in(\sqrt{3+2 \sqrt{2}}, 3)$. So, $y(t)$ is monotone increasing continuous function, and $\min y(t)=2 \sqrt{3+2 \sqrt{2}}-$ $\sqrt{2(\sqrt{2}+\sqrt{3+2 \sqrt{2}})} \approx 2.0613$. We get $2 t-\sqrt{t^{2}+2 t-3}-2-\sqrt{2} c \geq 0.0613-\sqrt{2} c>0$ (since the condition $c<(0.0613 / \sqrt{2}))$. Therefore, $z_{2}<\sigma_{2}$. On the other hand, $z_{2}-\sigma_{1}=$ $\sqrt{(2 a+\sqrt{4 a+1}-1) / 2 a^{2}}-(c / \sqrt{2} a)=(\sqrt{2 a+\sqrt{4 a+1}-1}-c) / \sqrt{2} a>0$, that is, $z_{2}>\sigma_{1}$. Thus, $\sigma_{1}<z_{2}<\sigma_{2}, \mathbf{y}_{2} \in B_{r}\left(\mathbf{x}^{*}\right)$, and $z_{2} \neq z^{*}$, that is, $\mathbf{y}_{2} \neq \mathbf{x}^{*}$. Let $\mathbf{x}_{0}=\mathbf{y}_{2}, \mathbf{x}_{1}=\mathbf{y}_{1}$; then, $F^{2}\left(\mathbf{x}_{0}\right)=\mathbf{x}^{*}$. Steps 2 and 3 complete the proof of condition (b).

Step 4. According to $D F\left(\mathbf{y}_{1}\right)=(\sqrt{4 a+1}-1) M \triangleq \omega M$, where $\omega=(\sqrt{4 a+1}-1)>0$, with Lemma 3.2, all eigenvalues of $D F\left(\mathbf{y}_{1}\right)$ lie in the interval $0<\omega(1-2 \epsilon) \leq \lambda_{j} \leq$ $\omega$. Thus, with Lemma 3.1, $\operatorname{det}\left[D F\left(\mathbf{y}_{1}\right)\right]=\prod_{j=1}^{N} \lambda_{j} \neq 0$. Moreover, according to $D F\left(\mathbf{y}_{2}\right)=$ $-\sqrt{4 a+2 \sqrt{4 a+1}-2} M \triangleq \Theta M$, where $\Theta=-\sqrt{4 a+2 \sqrt{4 a+1}-2}<0$, with Lemma 3.2, all eigenvalues of $D F\left(\mathbf{y}_{2}\right)$ lie in the interval $\Theta \leq \lambda_{j} \leq \Theta(1-2 \epsilon)<0$. Thus, with Lemma 3.1, $\operatorname{det}\left[D F\left(\mathbf{y}_{2}\right)\right]=\prod_{j=1}^{N} \lambda_{j} \neq 0$. Then, we have $F^{m}\left(\mathbf{x}_{0}\right)=\mathbf{x}^{*}$ and $\operatorname{det}\left[D F^{m}\left(\mathbf{x}_{0}\right)\right] \neq 0(m=2)$. Thus, condition (c) is complete. The system (1.1) has a snap-back repeller $\mathbf{x}^{*}$. Under the conditions of the Theorem 3.3, the DCML (1.1) is chaotic in the sense of Li-Yorke. The proof is completed.

\subsection{Numerical Simulation of Chaos}

When $N=300, a=1.8$, and $\epsilon=0.01$, the conditions of Theorem 3.3 are satisfied. The DCML (1.1) can be denoted as follows:

$$
\begin{gathered}
x_{n+1}(1)=(1-\epsilon)\left[1-a x_{n}^{2}(1)\right]+\epsilon\left[1-a x_{n}^{2}(2)\right], \\
x_{n+1}(2)=(1-\epsilon)\left(1-a x_{n}^{2}(2)\right)+\frac{\epsilon}{2}\left[1-a x_{n}^{2}(1)+1-a x_{n}^{2}(3)\right], \\
\vdots \\
x_{n+1}(299)=(1-\epsilon)\left(1-a x_{n}^{2}(299)\right)+\frac{\epsilon}{2}\left[1-a x_{n}^{2}(298)+1-a x_{n}^{2}(300)\right], \\
x_{n+1}(300)=\epsilon\left[1-a x_{n}^{2}(299)\right]+(1-\epsilon)\left[1-a x_{n}^{2}(300)\right] .
\end{gathered}
$$

The corresponding eigenvalues of $D F\left(\mathbf{x}^{*}\right)$ lie in the interval $(1-\sqrt{4 a+1},(1-\sqrt{4 a+1})(1-$ $2 \epsilon))$, that is, $\lambda_{i} \in(-1.8636,-1.8263)(i=1,2, \ldots, 300)$. These eigenvalues are strictly larger than one in absolute value. Starting from a random initial state, the number of iterations is 140. Simulation result is shown in Figure 1. When fixed $N=300, a=1.8$, and $\epsilon<0.0582$; 


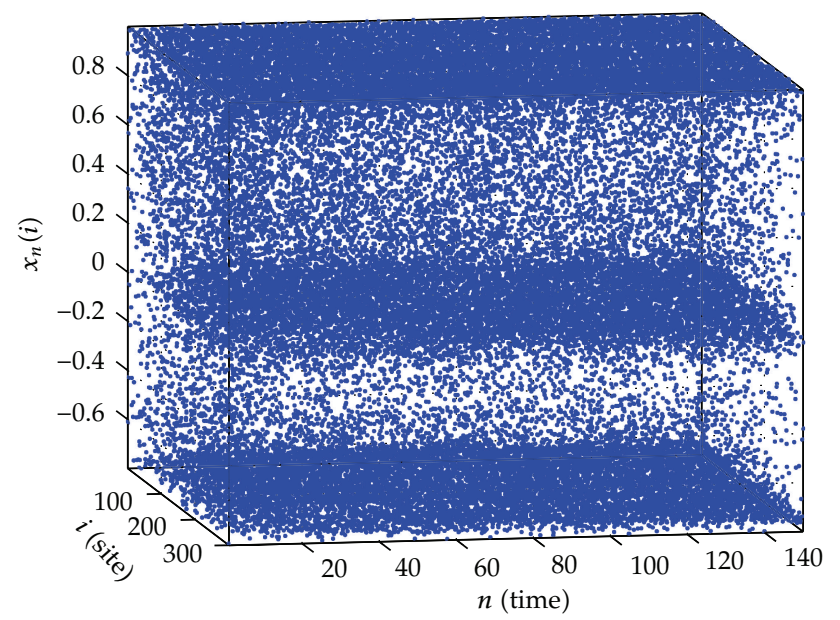

Figure 1: Spatiotemporal chaos in the DCML (3.18) without any control, with parameters $N=300, a=1.8$, $\epsilon=0.01$.

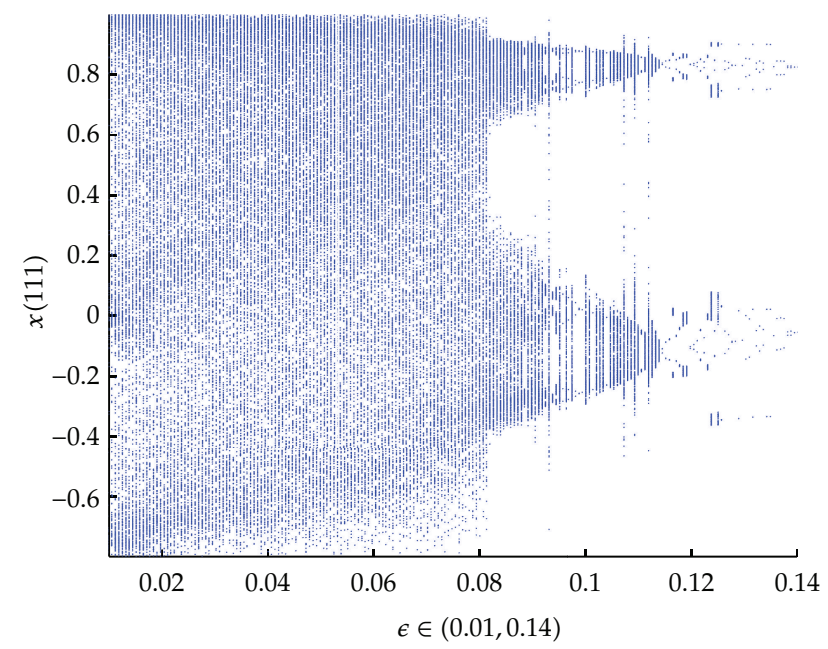

Figure 2: Bifurcation diagram of the DCML (3.18) versus $\epsilon \in(0.01,0.14)$ and $x(111)$, with initial point $(0.6, \ldots, 0.6)$.

these satisfy the conditions of Theorem 3.3. Thus, the system (3.18) should display chaotic behavior. The bifurcation diagram in Figure 2 also confirms the above statement.

\subsection{0-1 Test for Chaos in the DCML}

The 0-1 test for chaos was first reported in [31]. It and its modified version are applied directly to the time series data and do not require phase space reconstruction [31-36]. Moreover, the dimension and origin of the dynamical system are irrelevant. The $0-1$ test can efficiently distinguish chaotic behavior from regular (periodic or quasiperiodic) behavior in deterministic systems. The test result is 0 or 1 , depending on whether the dynamics is regular or chaotic, respectively. This method has been successfully applied to some typical 
chaotic systems [37-44] and experiment data [45]. We apply this method to the DCML. From another point of view, we show the existence of chaos in the DCML using the 0-1 test. Now, we describe the implementation of the $0-1$ test. The interested reader can consult [35] for further details. Consider discrete data sets $\phi(n)$ sampled at times $n=1,2,3, \ldots, N$, where $N$ is the total number of data points. $\phi(n)$ is an observable data from the underlying dynamic system.

Step 1. For a random number $c \in(\pi / 5,4 \pi / 5)$, define the translation variables

$$
p_{c}(n)=\sum_{j=1}^{n} \phi(j) \cos (j c), \quad q_{c}(n)=\sum_{j=1}^{n} \phi(j) \sin (j c) .
$$

Step 2. Define the mean square displacement $M_{c}(n)$ as follows:

$$
M_{c}(n)=\lim _{N \rightarrow \infty} \frac{1}{N} \sum_{j=1}^{N}\left[p_{c}(j+n)-p_{c}(j)\right]^{2}+\left[q_{c}(j+n)-q_{c}(j)\right]^{2}
$$

Note that this definition requires $n \ll N$. In practice, $n \leq N / 10$ yields good results. Denote $n_{\text {cut }}=\operatorname{round}(N / 10)$, where the function round $(x)$ rounds the elements of $x$ to the nearest integers.

Step 3. Define the modified mean square displacement

$$
D_{c}(n)=M_{c}(n)-V_{\mathrm{osc}}(c, n),
$$

where $V_{\text {osc }}(c, n)=(E \phi)^{2}(1-\cos n c) /(1-\cos c)$ and $E \phi=\lim _{N \rightarrow \infty}(1 / N) \sum_{j=1}^{N} \phi(j)$.

Step 4 . Form the vectors $\xi=\left(1,2, \ldots, n_{\text {cut }}\right)$ and $\Delta=\left(D_{c}(1), D_{c}(2), \ldots, D_{c}\left(n_{\text {cut }}\right)\right)$. Then define the correlation coefficient

$$
K_{c}=\operatorname{corr}(\xi, \Delta) \in[-1,1] .
$$

Step 5. Steps 1-4 are performed for $N_{c}$ values of $c$ chosen randomly in the interval $(\pi / 5,4 \pi / 5)$. In practice, $N_{c}=100$ is sufficient. We then compute the median of these $N_{c}$ values of $K_{c}$ to compute the final result $K=\operatorname{median}\left(K_{c}\right) . K \approx 0$ indicates regular dynamics, and $K \approx 1$ indicates chaotic dynamics.

Note that the $\left(p_{c}(n), q_{c}(n)\right)$-trajectories provide a direct visual test of whether the underlying dynamics is chaotic or nonchaotic. Namely, bounded trajectories in the $(p, q)$ plane imply regular dynamics, whereas Brownian-like (unbounded) trajectories imply chaotic dynamics [31,32]. With the sufficient length of the time series, $K \leq 0.1$ indicates that the dynamics is regular and $K>0.1$ indicates that the dynamics is chaotic [43].

Now, we apply the 0-1 test to the DCML (3.18). Fix $N=300, a=1.8$ and choose a random initial point $\left(x_{1}(1), x_{1}(2), \ldots, x_{1}(300)\right)$; we carry out the $0-1$ test with $\epsilon=0.03$ and $\epsilon=0.12$, respectively. Using the data set of $x(111)$ in the system (3.18), we get $K=0.9981$ at $\epsilon=0.03$ and $K=0.0030$ at $\epsilon=0.12$. The translation variables $(p, q)$ are shown in Figures 3 and 4 , respectively. 


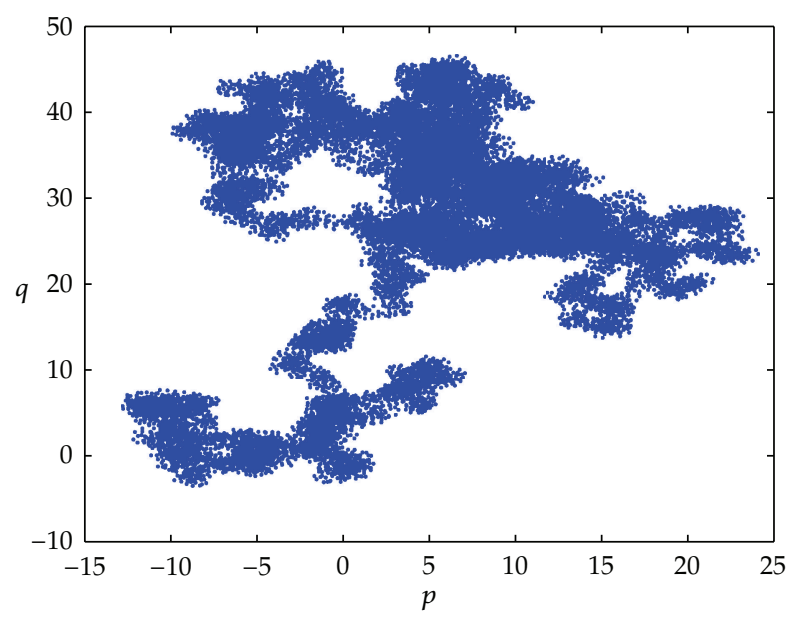

Figure 3: Plot of $p$ versus $q$ for the DCML (3.18) with $\epsilon=0.03$. We used 30000 data points of $x$ (111).

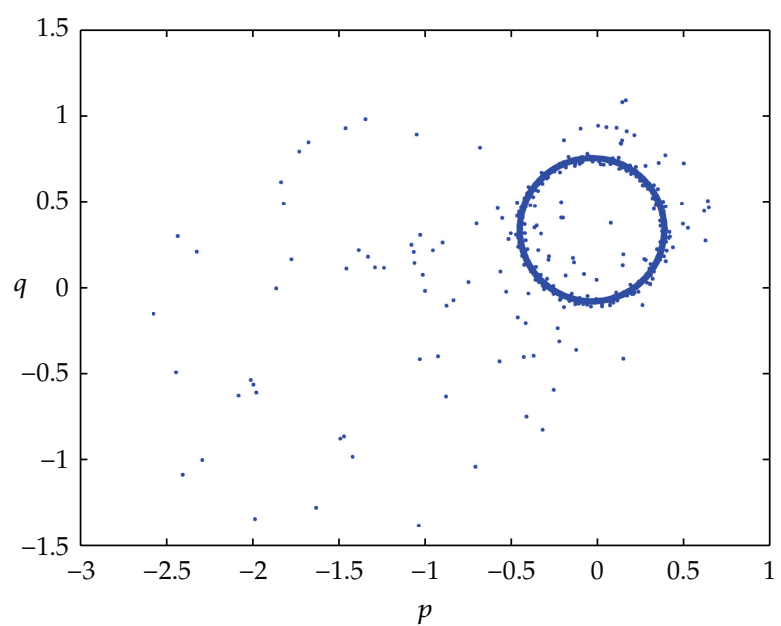

Figure 4: Plot of $p$ versus $q$ for the DCML (3.18) with $\epsilon=0.12$. We used 30000 data points of $x$ (111).

We take $N=300, a=1.8$ and let $\epsilon$ vary from 0.01 to 0.058 in increments of 0.01 . It is clear that the computed value of $K$ is effective for most values of $\epsilon$ in Figure 5. These 0-1 test results are consistent with numerical simulation in Section 3.2 and Theorem 3.3 in Section 3.1. Here we stress that the test results (chaos or nonchaos) are independent of the choices of initial point and changing the observable does not greatly alter the computed value of $K$.

\section{Control Spatiotemporal Chaos}

When $N=300, \epsilon=0.01$, and $a=1.8$, the system (3.18) displays chaotic dynamics. The DCML (3.18) has an unstable equilibrium point $X^{*}=[(\sqrt{4 a+1}-1) / 2 a, \ldots,(\sqrt{4 a+1}-1) / 2 a]^{T} \approx$ $[0.5177, \ldots, 0.5177]^{T}$. The goal of this section is to control spatiotemporal chaotic motions in 


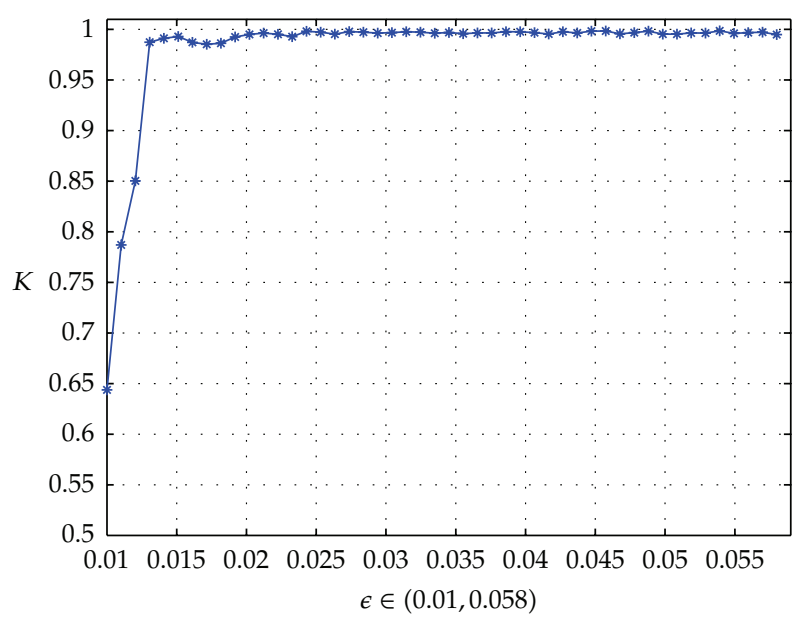

Figure 5: Plot of $K$ versus $\epsilon$ for the DCML (3.18) with $\epsilon \in(0.01,0.058)$ increased in increments of 0.01 . We used 30000 data points of $x(111)$.

the DCML (3.18) to the equilibrium point $X^{*}$ using delay feedback $[46,47]$. We rewrite the DCML (3.18) as

$$
X_{n+1}(i)=F\left(X_{n}(i), X_{n}(i-1), X_{n}(i+1)\right),
$$

where $X_{n}(i)=\left(x_{n}(1), x_{n}(2), \ldots, x_{n}(300)\right)^{T}$.

Theorem 4.1. With the local controllers $U_{n}(i)=\alpha_{1}\left[X_{n-1}(i)-F\left(X_{n}(i), X_{n}(i-1), X_{n}(i+1)\right)\right]$, the chaotic motion in the DCML (4.1) (i.e., (3.18)) can be controlled to the fixed point $X^{*}$, where $0.6407<\alpha_{1}<1$.

Proof. Since the local controllers are given by

$$
U_{n}(i)=\alpha_{1}\left[X_{n-1}(i)-F\left(X_{n}(i), X_{n}(i-1), X_{n}(i+1)\right)\right],
$$

we get the controlled DCML:

$$
\begin{aligned}
X_{n+1}(i) & =F\left(X_{n}(i), X_{n}(i-1), X_{n}(i+1)\right)+U_{n}(i) \\
& =\left(1-\alpha_{1}\right) F\left(X_{n}(i), X_{n}(i-1), X_{n}(i+1)\right)+\alpha_{1} X_{n-1}(i),
\end{aligned}
$$

where $\alpha_{1} \in(0.6407,1)$. Expanding $(4.3)$ around the fixed point $X^{*}$, we obtain

$$
X_{n+1}-X^{*}=\left.\frac{\partial F}{\partial X_{n}}\right|_{X^{*}}\left(X_{n}-X^{*}\right)+\left.\frac{\partial F}{\partial X_{n-1}}\right|_{X^{*}}\left(X_{n-1}-X^{*}\right) .
$$


Since $x_{n}(i) \approx f\left[x_{n-1}(i)\right], f(x)=1-a x^{2}$, and $x^{*}=(\sqrt{4 a+1}-1) / 2 a$, we have $x_{n}(i)-x^{*}=$ $\partial f /\left.\partial x_{n-1}\right|_{x^{*}}\left(x_{n-1}(i)-x^{*}\right)$. Thus, we get $x_{n-1}(i)-x^{*}=\left(1 /\left(\partial f /\left.\partial x_{n-1}\right|_{x^{*}}\right)\right)\left(x_{n}(i)-x^{*}\right)$ and

$$
X_{n-1}-X^{*}=\frac{1}{\partial f /\left.\partial x_{n-1}\right|_{x^{*}}}\left(X_{n}-X^{*}\right)
$$

Then, by using (4.4) and (4.5), we get

$$
\begin{aligned}
X_{n+1}-X^{*} & =\left.\frac{\partial F}{\partial X_{n}}\right|_{X^{*}}\left(X_{n}-X^{*}\right)+\left.\frac{\partial F}{\partial X_{n-1}}\right|_{X^{*}} \frac{1}{\partial f /\left.\partial x_{n-1}\right|_{x^{*}}}\left(X_{n}-X^{*}\right) \\
& =\left(\left.\frac{\partial F}{\partial X_{n}}\right|_{X^{*}}+\left.\frac{1}{\partial f /\left.\partial x_{n-1}\right|_{x^{*}}} \frac{\partial F}{\partial X_{n-1}}\right|_{X^{*}}\right)\left(X_{n}-X^{*}\right) .
\end{aligned}
$$

For the sake of simplicity, we denote $J$ by $\partial F /\left.\partial X_{n}\right|_{X^{*}}+\left.\left(1 /\left(\partial f /\left.\partial x_{n-1}\right|_{x^{*}}\right)\right)\left(\partial F / \partial X_{n-1}\right)\right|_{X^{*}} ;$ then

$$
J=\left(\begin{array}{ccccc}
\Lambda f^{\prime}(x(1)) & 2 \Theta f^{\prime}(x(2)) & 0 & \cdots & 0 \\
\Theta f^{\prime}(x(1)) & \Lambda f^{\prime}(x(2)) & \Theta f^{\prime}(x(3)) & \cdots & 0 \\
\vdots & \vdots & \ddots & \ddots & \vdots \\
0 & \cdots & \Theta f^{\prime}(x(298)) & \Lambda f^{\prime}(x(299)) & \Theta f^{\prime}(x(300)) \\
0 & \cdots & 0 & 2 \Theta f^{\prime}(x(299)) & \Lambda f^{\prime}(x(300))
\end{array}\right)+J_{2}
$$

where

$$
J_{2}=\left(\begin{array}{ccccc}
\frac{\alpha_{1}}{f^{\prime}(x(1))} & 0 & 0 & \ldots & 0 \\
0 & \frac{\alpha_{1}}{f^{\prime}(x(2))} & 0 & \ldots & 0 \\
\vdots & \vdots & \ddots & \ddots & \vdots \\
0 & \cdots & 0 & \frac{\alpha_{1}}{f^{\prime}(x(299))} & 0 \\
0 & \cdots & 0 & 0 & \frac{\alpha_{1}}{f^{\prime}(x(300))}
\end{array}\right),
$$

$\Theta=(\epsilon / 2)-\left(\epsilon \alpha_{1} / 2\right), \Lambda=1-\epsilon-\alpha_{1}+\alpha_{1} \epsilon$, and $f^{\prime}(x(i))=-2 a x(i)$. With the Gershgorin circle theorem (Lemma 3.2), we get

$$
\left|\lambda_{i}-\left[\left(1-\alpha_{1}\right)(1-\epsilon) f^{\prime}(x(i))+\frac{\alpha_{1}}{f^{\prime}(x(i))}\right]\right|<\left(1-\alpha_{1}\right) \epsilon f^{\prime}(x(i)),
$$

that is,

$$
\left(1-\alpha_{1}\right) f^{\prime}(x(i))-2 \epsilon\left(1-\alpha_{1}\right) f^{\prime}(x(i))+\frac{\alpha_{1}}{f^{\prime}(x(i))}<\lambda_{i}<\left(1-\alpha_{1}\right) f^{\prime}(x(i))+\frac{\alpha_{1}}{f^{\prime}(x(i))} .
$$

Solving inequality (4.10), we obtain $-1.8263+1.2897 \alpha_{1}<\lambda_{i}<-1.8636+1.3270 \alpha_{1}$. Since $0.6407<\alpha_{1}<1$, we get $\left|\lambda_{i}\right|<1$. The proof is completed. 


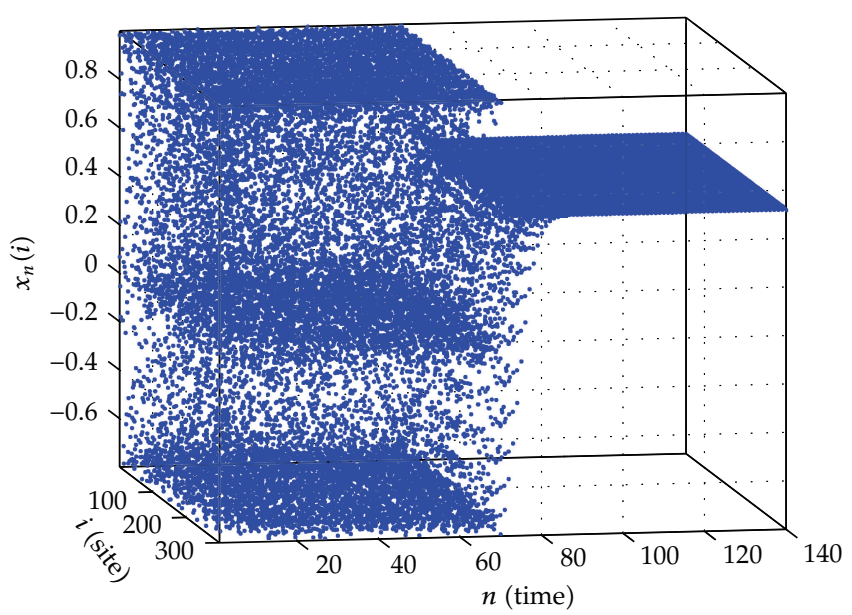

Figure 6: The control result of the DCML (3.18), with the parameter values $a=1.8, \epsilon=0.01$, and $\alpha_{1}=0.85$. The feedback control starts at the 71st iteration.

The simulation result is shown in Figure 6. Chaotic motions are quickly controlled to the fixed point $X^{*} \approx[0.5177, \ldots, 0.5177]^{T}$.

Remark 4.2. In the process of proving Theorems 3.3 and 4.1, we only need to know that eigenvalues are greater (or less) than one in absolute and it is not necessary to compute explicitly the eigenvalues. These ideas avoid difficulties in calculating eigenvalues in higherdimension DCMLs using the Gershgorin circle theorem.

\section{Conclusion}

With the Marotto theorem and the Gershgorin circle theorem, we have theoretically analyzed the chaos in the DCML with open boundary conditions, which presents a theoretical foundation for chaos analysis of the DCML. What is more is that the 0-1 test further confirms the existence of chaos and we control spatiotemporal chaotic motions in the DCML to period-1 orbits. Stability analysis is presented. The results of simulations are consistent with theoretical analysis. We wish to emphasize that the methods of this paper can be used in all those cases where the eigenvalues of Jacobi matrix are difficult to calculate in CMLs.

\section{Acknowledgment}

The paper was supported by NSFC (no. 10871074).

\section{References}

[1] K. Kaneko, Theory and Applications of Coupled Map Lattices, Nonlinear Science: Theory and Applications, John Wiley \& Sons, New York, NY, USA, 1993.

[2] A. Jakobsen, "Symmetry breaking bifurcations in a circular chain of $N$ coupled logistic maps," Physica D, vol. 237, no. 24, pp. 3382-3390, 2008.

[3] J. Y. Chen, K. W. Wong, H. Y. Zheng, and J. W. Shuai, "The coupling of dynamics in coupled map lattices," Discrete Dynamics in Nature and Society, vol. 7, no. 3, pp. 157-160, 2002. 
[4] M. K. Ali and J. Q. Fang, "Synchronization of spatiotemporal chaos using nonlinear feedback functions," Discrete Dynamics in Nature and Society, vol. 1, pp. 179-184, 1997.

[5] J. Q. Fang and M. K. Ali, "Nonlinear feedback control of spatiotemporal chaos in coupled map lattices," Discrete Dynamics in Nature and Society, vol. 1, pp. 283-305, 1998.

[6] A. V. Guedes and M. A. Savi, "Spatiotemporal chaos in coupled logistic maps," Physica Scripta, vol. 81, no. 4, Article ID 045007, 2010.

[7] K. Wang, W. Pei, Y. M. Cheung, Y. Shen, and Z. He, "Estimation of chaotic coupled map lattices using symbolic vector dynamics," Physics Letters, Section A, vol. 374, no. 4, pp. 562-566, 2010.

[8] G. Chen and S. T. Liu, "On generalized synchronization of spatial chaos," Chaos, Solitons and Fractals, vol. 15, no. 2, pp. 311-318, 2003.

[9] W.-W. Lin and Y. Q. Wang, "Chaotic synchronization in coupled map lattices with periodic boundary conditions," SIAM Journal on Applied Dynamical Systems, vol. 1, no. 2, pp. 175-189, 2002.

[10] W.-W. Lin, C.-C. Peng, and Y.-Q. Wang, "Chaotic synchronization in lattices of two-variable maps coupled with one variable," IMA Journal of Applied Mathematics, vol. 74, no. 6, pp. 827-850, 2009.

[11] K. E. Zhu and T. Chen, "Controlling spatiotemporal chaos in coupled map lattices," Physical Review E, vol. 63, no. 6, pp. 067201/1-067201/4, 2001.

[12] W. Huang, "On the stabilization of internally coupled map lattice systems," Discrete Dynamics in Nature and Society, no. 2, pp. 345-356, 2004.

[13] P. Li, Z. Li, W. A. Halang, and G. Chen, "Li-Yorke chaos in a spatiotemporal chaotic system," Chaos, Solitons and Fractals, vol. 33, no. 2, pp. 335-341, 2007.

[14] P. Li, Z. Li, and W. A. Halang, "Chaotification of spatiotemporal systems," International Journal of Bifurcation and Chaos in Applied Sciences and Engineering, vol. 20, no. 7, pp. 2193-2202, 2010.

[15] C. Tian and G. Chen, "Chaos in the sense of Li-Yorke in coupled map lattices," Physica A, vol. 376, pp. 246-252, 2007.

[16] M. F. Shen, L. X. Lin, X. Y. Li, and C. Q. Chang, "Initial condition estimate of coupled map lattices system based on symbolic dynamics," Acta Physica Sinica, vol. 58, no. 5, pp. 2921-2929, 2009 (Chinese).

[17] I. Aranson, D. Golomb, and H. Sompolinsky, "Spatial coherence and temporal chaos in macroscopic systems with asymmetrical couplings," Physical Review Letters, vol. 68, no. 24, pp. 3495-3498, 1992.

[18] Y. Shi and P. Yu, "On chaos of the logistic maps," Dynamics of Continuous, Discrete E Impulsive Systems: Series B, vol. 14, no. 2, pp. 175-195, 2007.

[19] Y. Shi and G. Chen, "Discrete chaos in Banach spaces," Science in China: Series A, vol. 48, no. 2, pp. 222-238, 2005.

[20] R. M. May, "Simple mathematical models with very complicated dynamics," Nature, vol. 261, no. 5560, pp. 459-467, 1976.

[21] M. W. Hirsch, S. Smale, and R. L. Devaney, Differential Equations, Dynamical Systems, and an Introduction to Chaos, vol. 60 of Pure and Applied Mathematics, Academic Press, Amsterdam, The Netherlands, 2nd edition, 2004.

[22] F. R. Marotto, "Snap-back repellers imply chaos in $R^{n}$," Journal of Mathematical Analysis and Applications, vol. 63, no. 1, pp. 199-223, 1978.

[23] F. R. Marotto, “On redefining a snap-back repeller," Chaos, Solitons and Fractals, vol. 25, no. 1, pp. 25-28, 2005.

[24] T. Y. Li and J. A. Yorke, "Period three implies chaos," The American Mathematical Monthly, vol. 82, no. 10, pp. 985-992, 1975.

[25] C. P. Li and G. Chen, "An improved version of the Marotto theorem," Chaos, Solitons and Fractals, vol. 18, no. 1, pp. 69-77, 2003.

[26] G. Chen, S.-B. Hsu, and J. Zhou, "Snapback repellers as a cause of chaotic vibration of the wave equation with a van der Pol boundary condition and energy injection at the middle of the span," Journal of Mathematical Physics, vol. 39, no. 12, pp. 6459-6489, 1998.

[27] M. W. Hirsch and S. Smale, Differential Equations, Dynamical Systems, and Linear Algebra, Academic Press, New York, NY, USA, 1974.

[28] C.-C. Peng, "Numerical computation of orbits and rigorous verification of existence of snapback repellers," Chaos, vol. 17, no. 1, p. 013107, 2007.

[29] R. L. Burden and D. J. Faires, Numerical Analysis, Brooks Cole Press, Boston, Mass, USA, 1993.

[30] C. Meyer, Matrix Analysis and Applied Linear Algebra, Society for Industrial and Applied Mathematics (SIAM), Philadelphia, Pa, USA, 2000. 
[31] G. A. Gottwald and I. Melbourne, "A new test for chaos in deterministic systems," Proceedings of The Royal Society of London, vol. 460, no. 2042, pp. 603-611, 2004.

[32] G. A. Gottwald and I. Melbourne, "Testing for chaos in deterministic systems with noise," Physica D, vol. 212, no. 1-2, pp. 100-110, 2005.

[33] J. Hu, W. W. Tung, J. Gao, and Y. Cao, "Reliability of the 0-1 test for chaos," Physical Review E, vol. 72, no. 5, Article ID 056207, pp. 1-5, 2005.

[34] G. A. Gottwald and I. Melbourne, "Comment on 'reliability of the 0-1 test for chaos'," Physical Review E, vol. 77, no. 2, Article ID 028201, 2008.

[35] G. A. Gottwald and I. Melbourne, "On the implementation of the 0-1 test for chaos," SIAM Journal on Applied Dynamical Systems, vol. 8, no. 1, pp. 129-145, 2009.

[36] G. A. Gottwald and I. Melbourne, "On the validity of the 0-1 test for chaos," Nonlinearity, vol. 22, no. 6, pp. 1367-1382, 2009.

[37] G. Litak, A. Syta, and M. Wiercigroch, "Identification of chaos in a cutting process by the 0-1 test," Chaos, Solitons and Fractals, vol. 40, no. 5, pp. 2095-2101, 2009.

[38] G. Litak, A. Syta, M. Budhraja, and L. M. Saha, "Detection of the chaotic behaviour of a bouncing ball by the 0-1 test," Chaos, Solitons and Fractals, vol. 42, no. 3, pp. 1511-1517, 2009.

[39] Y. Kim, "Identification of dynamical states in stimulated Izhikevich neuron models by using a 0-1 test," Journal of the Korean Physical Society, vol. 57, no. 6, pp. 1363-1368, 2010.

[40] C. W. Kulp and S. Smith, "Characterization of noisy symbolic time series," Physical Review E, vol. 83, no. 2, Article ID 026201, 2011.

[41] M. Romero-Bastida, M. A. Olivares-Robles, and E. Braun, "Probing Hamiltonian dynamics by means of the 0-1 test for chaos," Journal of Physics A, vol. 42, no. 49, p. 495102, 2009.

[42] M. Romero-Bastida and A. Y. Reyes-Martnez, "Efficient time-series detection of the strong stochasticity threshold in Fermi-Pasta-Ulam oscillator lattices," Physical Review E, vol. 83, no. 1, Article ID 016213, 2011.

[43] K. H. Sun, X. Liu, and C. X. Zhu, "The 0-1 test algorithm for chaos and its applications," Chinese Physics B, vol. 19, no. 11, Article ID 110510, 2010.

[44] D. Cafagna and G. Grassi, "An effective method for detecting chaos in fractional-order systems," International Journal of Bifurcation and Chaos, vol. 20, no. 3, pp. 669-678, 2010.

[45] I. Falconer, G. A. Gottwald, I. Melbourne, and K. Wormnes, "Application of the 0-1 test for chaos to experimental data," SIAM Journal on Applied Dynamical Systems, vol. 6, no. 2, pp. 395-402, 2007.

[46] X. S. Luo and B. H. Wang, "Controlling spatiotemporal chaos in coupled map lattice by delay feedback," Atomic Energey Science and Technology, vol. 35, pp. 56-59, 2001 (Chinese).

[47] K. Konishi and H. Kokame, "Decentralized delayed-feedback control of a one-way coupled ring map lattice," Physica D, vol. 127, no. 1-2, pp. 1-12, 1999. 


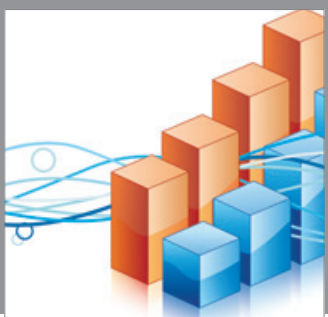

Advances in

Operations Research

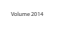

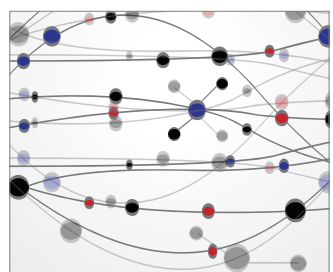

\section{The Scientific} World Journal
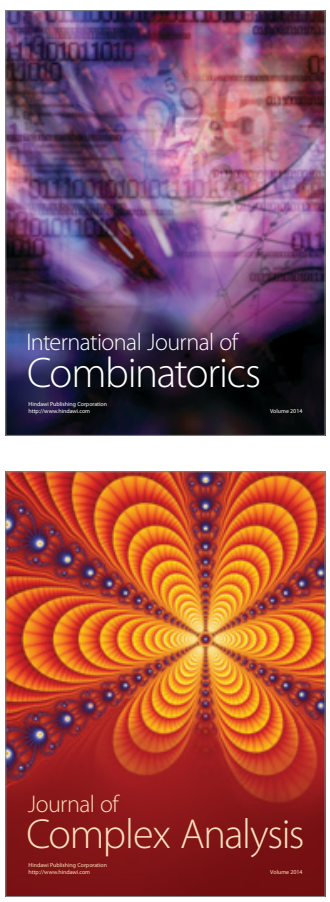

International Journal of

Mathematics and

Mathematical

Sciences
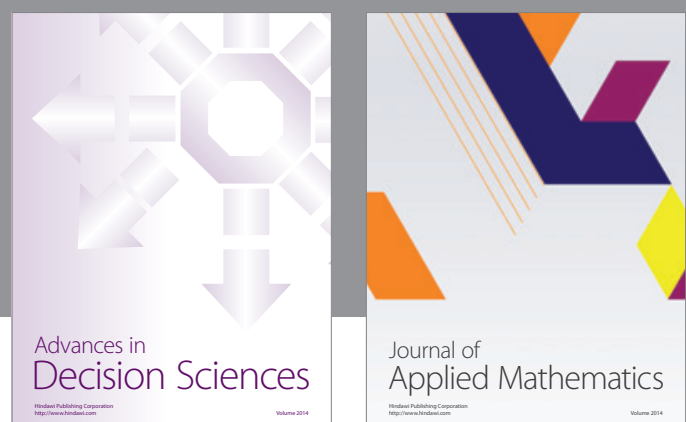

Journal of

Applied Mathematics
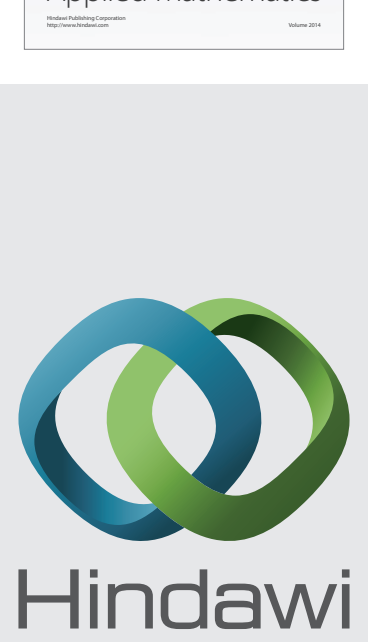

Submit your manuscripts at http://www.hindawi.com
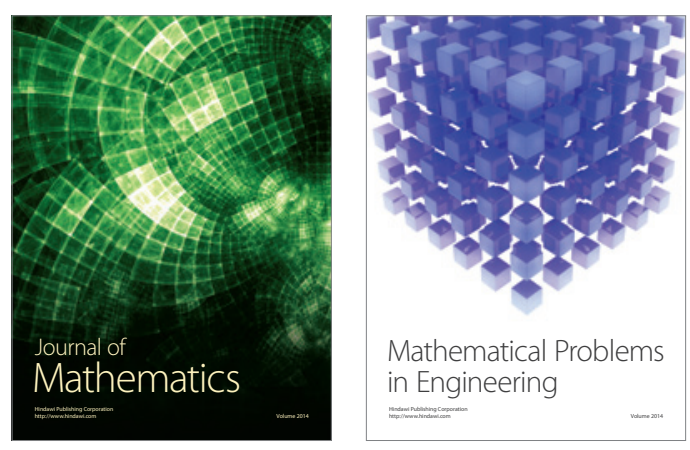

Mathematical Problems in Engineering
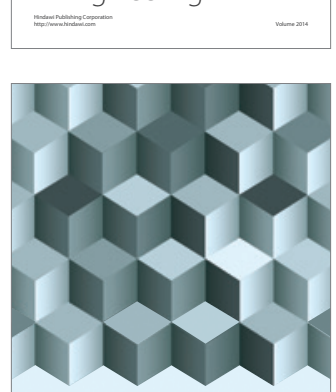

Journal of

Function Spaces
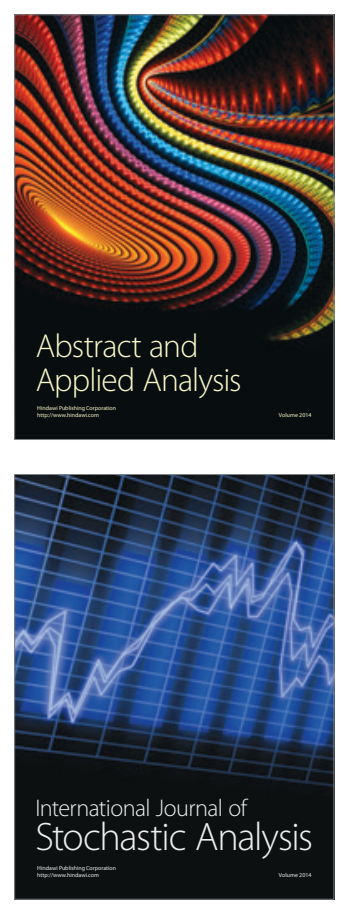

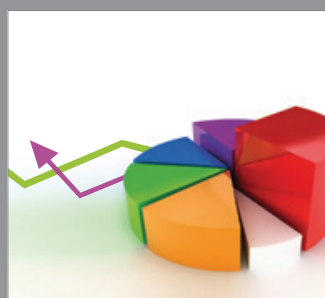

ournal of

Probability and Statistics

Promensencen
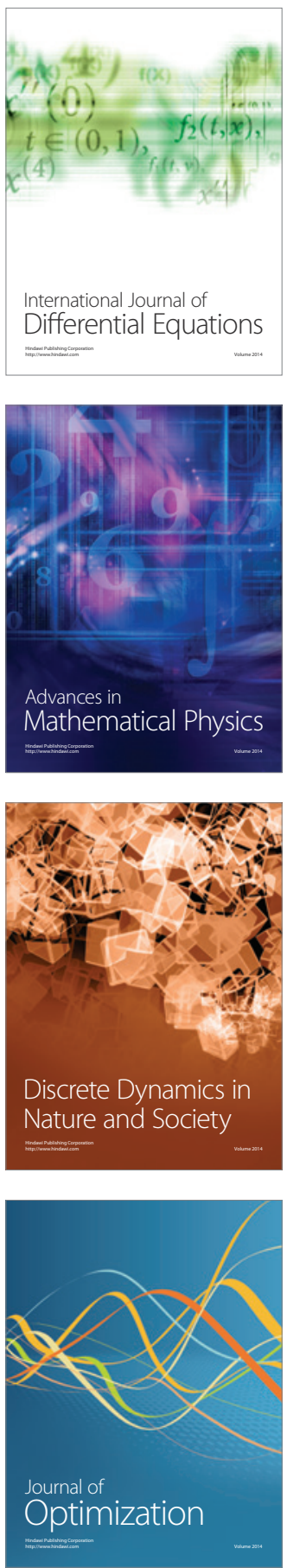